\title{
Severe and protracted cholestasis in 44 young men taking bodybuilding supplements: assessment of genetic, clinical and chemical risk factors
}

\author{
Andrew Stolz ${ }^{1}$ (D) | Victor Navarro ${ }^{2} \mid$ Paul H. Hayashi $^{3}$ | Robert J. Fontana ${ }^{4}$ | Huiman \\ X. Barnhart ${ }^{5}$ | Jiezhun Gu ${ }^{5}$ | Naga P. Chalasani ${ }^{6}$ (D) | Maricruz M. Vega ${ }^{2}$ | Herbert \\ L. Bonkovsky ${ }^{7}$ | Leonard B. Seeff ${ }^{2}$ | Jose Serrano ${ }^{8}$ | Bharathi Avula ${ }^{9}$ | Ikhlas \\ A. Khan ${ }^{9}$ | Elizabeth T. Cirulli ${ }^{5}$ | David E. Kleiner ${ }^{10}$ | Jay H. Hoofnagle ${ }^{8}$ | for the DILIN \\ Investigators*
}

${ }^{1}$ University of Southern California, Los Angeles, California

${ }^{2}$ Einstein Medical Center, Philadelphia, Pennsylvania

${ }^{3}$ University of North Carolina, Chapel Hill, North Carolina

${ }^{4}$ University of Michigan, Ann Arbor, Michigan

${ }^{5}$ Duke University, Durham, North Carolina ${ }^{6}$ Indiana University School of Medicine, Indianapolis, Indiana

${ }^{7}$ Wake Forest University School of Medicine, Winston-Salem, North Carolina

${ }^{8}$ Liver Diseases Research Branch, National Institute of Diabetes and Digestive and Kidney Diseases, National Institutes of Health, Bethesda, Maryland

${ }^{9}$ National Center for Natural Product Research, School of Pharmacy, University of Mississippi, University City, Mississippi

${ }^{10}$ Laboratory of Pathology, National Cancer Institute, Bethesda, Maryland

Correspondence

Dr. Andrew Stolz, Keck School of Medicine of USC, Los Angeles, CA.

Email: astolz@usc.edu

Funding information

The DILIN Network is structured as a U01 cooperative agreement with funds provided by the National Institute of Diabetes and Digestive and Kidney Diseases (NIDDK) under grants: 2U01-DK065176-10 (Duke), 2U01-DK065201-15 (UNC), 2U01-

DK065184-15 (Michigan), 2U01-DK065211-

\section{Summary}

Background: Bodybuilding supplements can cause a profound cholestatic syndrome. Aim: To describe the drug-Induced liver injury network's experience with liver injury due to bodybuilding supplements.

Methods: Liver injury pattern, severity and outcomes, potential genetic associations, and exposure to anabolic steroids by product analysis were analysed in prospectively enrolled subjects with bodybuilding supplement-induced liver injury with causality scores of probable or higher.

Results: Forty-four males (mean age 33 years) developed liver injury with a median latency of 73 days. Forty-one per cent presented with hepatocellular pattern of liver injury as defined by the $R>5$ ([Fold elevation of ALT] $\div$ [Fold elevation of Alk Phos] (mean, range $=6.4,0.5-31.4, \mathrm{n}=42$ ) despite all presenting with clinical features of cholestatic liver injury (100\% with jaundice and $84 \%$ with pruritus). Liver biopsy (59\% of subjects) demonstrated a mild hepatitis and profound cholestasis in most without bile duct injury, loss or fibrosis. Seventy-one per cent were hospitalised, and none died or required liver transplantation. In some, chemical analysis revealed anabolic steroid controlled substances not listed on the label. No enrichment of genetic variants associated with cholestatic syndromes was found, although mutations in ABCB11 (present in up to 20\%) were significantly different than in ethnically matched controls.

Conclusions: Patients with bodybuilding supplements liver injury uniformly presented with cholestatic injury, which slowly resolved. The ingested products often contained anabolic steroids not identified on the label, and no enrichment in genetic variants was found, indicating a need for additional studies.

${ }^{*}$ The DILIN Investigators are listed in Appendix S1.

The Handling Editor for this article was Professor Stephen Harrison, and it

was accepted for publication after full peer-review. 
15 (Indiana), 1U01-DK083027-09 (TJH/ UPenn), 1U01-DK082992-05 (Mayo), 1U01DK083020-10 (USC). Additional funding is provided by CTSA grants: UL1 RR025761 (Indiana), UL1 TR000083 (UNC), UL1 RR024986 (Michigan), UL1 RR TR001855 (USC) and in part by the Intramural Research Program of the NIH, National Cancer Institute.

\section{1 | INTRODUCTION}

Androgenic anabolic steroids are modified androgens or prohormones that promote muscle growth and are used to enhance athletic performance or improve physique. ${ }^{1,2}$ Several anabolic steroids have been approved as therapy of hypogonadism, cachexia, anaemia and other disorders. However, these products can also cause diverse liver abnormalities, including severe and prolonged cholestatic liver injury, peliosis hepatis, hepatic adenoma and even hepatocellular carcinoma with prolonged use, albeit in a minority of patients. ${ }^{3-7}$ These risks, as well as cardiovascular injury, hyperlipidaemia, and infertility, along with the development of dependence, have prompted increasing Food and Drug Administration and Drug Enforcement Administration (DEA) regulation of anabolic steroids in the United States. Despite the increased oversight, numerous examples of contamination of bodybuilding supplements with illegal anabolic steroids have been reported, demonstrating that these supplements remain an important source of illicit anabolic steroid exposure. ${ }^{3,8-10}$

Little is known about the pathophysiological mechanisms of prolonged cholestasis associated with anabolic steroids or who is at risk for developing liver injury. Previous small studies have identified a few variants associated with genetic cholestatic disorders in those that develop cholestastic liver injury after using bodybuilding supplements. ${ }^{11,12}$ Anabolic steroid modifications allowing for oral administration and those specifically with a C-17 alkylated modification are known to be associated with cholestatic liver injury, ${ }^{1}$ but a detailed time course of liver injury, histologic evaluation, genetic characterisation and chemical analysis of supplements in a large cohort is lacking. The drug-induced liver injury network (DILIN) has prospectively enrolled patients with drug or herbal and dietary supplement-associated liver injury to characterise their clinical features and to generate a well-phenotyped biospecimen repository for future mechanistic and genetic association studies. ${ }^{13,14} \mathrm{~A}$ previous review of the DILIN's experience with herbal and dietary supplement, which constituted the second most common class of agents causing drug-induced liver injury (DILI), identified supplements advertised to promote bodybuilding as the largest class within this category. ${ }^{15}$ Patients consuming bodybuilding supplements often presented with a distinct clinical phenotype of a profound cholestatic liver injury in the absence of significant biliary obstruction. The current report now describes the clinical features, laboratory findings, histological features and outcomes among 44 consecutive patients enrolled with bodybuilding supplements induced liver injury in this well-characterised prospective cohort of patients. In addition, chemical analysis of the ingested products in 14 subjects and potential genetic associations were conducted in 41 cases, respectively, to explore potential exposure to anabolic steroids and mechanisms of liver injury.

\section{MATERIALS AND METHODS}

\subsection{Recruitment of DILIN prospective study}

All patients were enrolled into an ongoing, multicentre prospective Registry study which had been approved by Institutional Review Boards at all clinical sites and the data coordinating centre, as well as by an independent Data and Safety Monitoring Board appointed by the NIDDK. All subjects (NCT00345930) provided written informed consent for the collection and analysis of clinical data and biological specimens for genetic testing. ${ }^{13,14}$ The DILIN previously reported its experience with liver injury due to herbal and dietary supplement between 2004 and March 2013. ${ }^{15}$ Reassessment of these original herbal and dietary supplement cases identified 44 attributed to bodybuilding supplements. For the study, liver injury onset was defined as the first day of documented liver injury after being exposed to the bodybuilding supplement and meeting one of the following criteria for inclusion into the study on two successive blood samples, collected at least $24 \mathrm{~h}$ apart: (a) a serum aspartate aminotransferase (AST) or alanine aminotransferase (ALT) level greater than five times the upper limit of normal (or five times the baseline elevated value if known); (b) a serum alkaline phosphatase (Alk Phos) level that exceeded two times the Upper limit of normal (ULN; or two times a pretreated abnormal baseline value if known); or (c) a serum total bilirubin of $2.5 \mathrm{mg} / \mathrm{dL}$ or greater or an international normalised ratio (INR) greater than 1.5 in combination with any enzyme elevation. Patients with injury within the past 6 months were eligible for enrolment into the prospective study. A detailed review of the patient's medical history and record was performed at initial enrolment, and narrative generated summarising key clinical features. Additional laboratory tests and imaging studies were performed to exclude other potential aetiologies. In addition, patients were screened for hepatitis A, B, C, E, HIV, CMV and EBV infections and for routine autoantibodies. Patients were asked to return for follow-up at 6 months, and if evidence of persistent liver injury was noted at that time, they were asked to return for further follow-up at 12 and 24 months. 
The assessment of causality between the liver injury and the implicated agent was carried out using a standardised process based upon the pertinent clinical, biochemical, radiological and histologic findings if available after the 6-month follow-up visit. ${ }^{16}$ DILIN expert opinion causality scores ranged from 1 (definite: $\geq 95 \%$ likelihood), 2 (highly likely: 75\%-94\% likelihood), 3 (probable: 50\%-74\% likelihood), 4 (possible $25 \%-49 \%$ likelihood) to 5 (unlikely: $<25 \%$ likelihood). The pattern of liver injury was defined by the $R=[$ ALT/ULN] $\div$ [Alk Phos/ ULN], based on which cases were defined as hepatocellular $(R>5)$, cholestatic $(R<2)$ or mixed $(R=2-5)$. The severity of injury was scored from mild (1), moderate (2), moderate-hospitalised (3), severe (4) and fatal (5), if death from liver disease or liver transplantation occurred within 6 months of the onset. ${ }^{14}$

\section{2 | Liver histopathology}

There was no requirement for a liver biopsy for enrolment, but if performed for routine clinical care, available slides were collected and sent to a single expert liver histopathologist (DEK) who examined and scored them for multiple histological features as well for a global pattern of liver injury. ${ }^{17}$

\section{3 | Anabolic steroid analysis}

Chemical analysis of bodybuilding supplements products for botanicals, amino acids, vitamins, pharmaceutical agents and 37 different anabolic steroids were performed using ultra-high performance liquid chromatography-quadrupole time of flight-mass spectrometry (UHPLC/QToF-MS) with modifications in the extraction methodology (see Data S1).

\section{4 | DNA sequencing and genotyping}

Genomic DNA samples from 25 subjects with bodybuilding supplements DILI underwent whole genome sequencing (WGS) at Duke University using Illumina GAllx or HiSeq 2000 or 2500 sequencers. An additional 16 cases were genotyped with an exome chip: 10 cases were genotyped with the Illumina HumanExome chip, and six cases were genotyped with the Illumina Multi-Ethnic Genotyping Array (MEGA), which includes the same low-frequency coding variants as the HumanExome chip. The genes $A B C B 11$ (BSEP), ABCB4 (MDR3) and ATP8B1 (PFIC 1) were chosen as candidate genes as they are known to cause progressive familial intrahepatic cholestasis. ${ }^{18}$ Data were analysed according to standard protocols as described in the Supplemental Methods.

\section{5 | Data collection and statistics}

Data from cases were deposited in electronic case report forms (eCRFs) via the InForm ${ }^{\mathrm{TM}}$ (Oracle Health Solutions, CA) and were downloaded in 2016. Summary statistics were reported as median with range for continuous data and frequency with percentage for categorical data using the SAS 9.4 (SAS Institute, Cary, NC) statistical software.

\section{RESULTS}

\subsection{Demographics, clinical features and laboratory findings}

The demographic and clinical features of the 44 patients with liver injury attributed to bodybuilding supplements enrolled between 2004 and November 2013 are shown in Table 1 and laboratory and outcome features in Table 2. All patients were adult males with a median age of 31.5 years (range: $21-59$ ) with $82 \%$ self-reporting their race as white or Caucasian, $11 \%$ as African-Americans, 2\% Asian and 5\% multiracial or other and 11\% identified themselves as Latino. Twenty-one per cent reported prior drug allergies and $80 \%$ admitted to drinking alcohol at least once in the past year. Of those that consumed alcohol, five were heavy drinkers as defined by consuming more than three drinks per day. ${ }^{19}$ There were no significant

TABLE 1 Clinical and demographic features of 44 patients with suspected anabolic steroid jaundice

\begin{tabular}{|c|c|c|}
\hline Feature & $\begin{array}{l}\text { Number } \\
\text { positive } \\
\text { or median }\end{array}$ & $\begin{array}{l}\text { Proportion } \\
\text { or range }\end{array}$ \\
\hline Sex (male) & 44 & $100 \%$ \\
\hline Age (yr) & 31.5 & $21-59$ \\
\hline \multicolumn{3}{|l|}{ Self-reported race } \\
\hline White/Caucasian & 36 & $82 \%$ \\
\hline Black/African American & 5 & $11 \%$ \\
\hline Asian-American & 1 & $2 \%$ \\
\hline Other/Multiracial & 2 & $5 \%$ \\
\hline Weight $(\mathrm{kg})(\mathrm{n}=43)^{\mathrm{a}}$ & 88.2 & $50.5-117.1$ \\
\hline $\mathrm{BMI}\left(\mathrm{kg} / \mathrm{m}^{2}\right)(\mathrm{n}=43)^{\mathrm{a}}$ & 26.2 & 19.9-37.4 \\
\hline \multicolumn{3}{|l|}{ Pre-existing conditions } \\
\hline Diabetes/endocrine Ds & 0 & $0 \%$ \\
\hline Alcohol use $(n=43)^{a}$ & 35 & $81 \%$ \\
\hline HCV RNA positive $(n=43)^{a}$ & 3 & $8 \%$ \\
\hline HIV positive & 1 & $2 \%$ \\
\hline Family history liver disease & 1 & $2 \%$ \\
\hline Bodybuilding supplement latency (mo) & 2.5 & $0.45-15.5$ \\
\hline \multicolumn{3}{|l|}{ Signs and symptoms } \\
\hline Jaundice & 44 & $100 \%$ \\
\hline Pruritus & 37 & $84 \%$ \\
\hline Abdominal pain & 26 & $59 \%$ \\
\hline Nausea & 27 & $61 \%$ \\
\hline Rash & 11 & $25 \%$ \\
\hline Fever & 7 & $16 \%$ \\
\hline
\end{tabular}

${ }^{\text {aD }}$ ata not available on all 44 patients; number given in brackets. 
TABLE 2 Laboratory features of 44 patients with suspected anabolic steroid jaundice

\begin{tabular}{|c|c|c|}
\hline Feature & $\begin{array}{l}\text { Number positive } \\
\text { or median }\end{array}$ & $\begin{array}{l}\text { Proportion } \\
\text { or range }\end{array}$ \\
\hline \multicolumn{3}{|l|}{ Initial values at onset } \\
\hline $\mathrm{ALT}(\mathrm{U} / \mathrm{L})$ & 169 & 61-1254 \\
\hline AST (U/L) & 82 & $38-278$ \\
\hline Alk Phos (U/L) & 111 & $53-461$ \\
\hline Bilirubin (mg/dL) & 9.8 & 4.1-45.7 \\
\hline \multicolumn{3}{|l|}{$R$ value } \\
\hline \multicolumn{3}{|l|}{ Injury pattern $(n=42)$} \\
\hline Cholestatic & 12 & $29 \%$ \\
\hline Mixed & 13 & $31 \%$ \\
\hline Hepatocellular & 17 & $41 \%$ \\
\hline \multicolumn{3}{|l|}{ Peak values during course } \\
\hline ALT (U/L) & 189 & $64-1254$ \\
\hline AST (U/L) & 128 & $55-472$ \\
\hline Alk Phos (U/L) & 257 & $83-625$ \\
\hline Bilirubin (mg/dL) & 25.8 & $7.3-63.0$ \\
\hline INR & 1.1 & $0.9-2.1$ \\
\hline \multicolumn{3}{|l|}{ Duration of abnormalities } \\
\hline Bilirubin $\geq 2.5 \mathrm{mg} / \mathrm{dL}^{\mathrm{b}}(\mathrm{n}=36)$ & 94.3 & $34-459$ \\
\hline \multicolumn{3}{|c|}{ Chronic laboratory abnormalities (mo) ${ }^{a}$} \\
\hline $6(n=31)^{c}$ & 4 & $39 \%^{c}$ \\
\hline $12(n=12)$ & 2 & $50 \%^{\mathrm{d}}$ \\
\hline $24(n=2)$ & 2 & $100 \%^{d}$ \\
\hline \multicolumn{3}{|l|}{ Other Investigations or interventions } \\
\hline MRCP or ERCP & 9 & $20 \%$ \\
\hline Liver biopsy & 22 & $50 \%$ \\
\hline Corticosteroid therapy $(n=43)$ & 7 & $16 \%$ \\
\hline $\begin{array}{l}\text { Ursodiol therapy at time of } \\
\text { enrollment }\end{array}$ & 21 & $47 \%$ \\
\hline Creatinine $>1.5 \mathrm{mg} / \mathrm{dL}$ & 6 & $14 \%$ \\
\hline \multicolumn{3}{|l|}{ Causality, severity and outcomes } \\
\hline \multicolumn{3}{|l|}{ Severity score } \\
\hline 1 (mild, anicteric) & 0 & $0 \%$ \\
\hline 2 (moderate, icteric) & 10 & $23 \%$ \\
\hline 3 (moderate, hospitalised) & 25 & $57 \%$ \\
\hline 4 (severe, organ failure) & 9 & $21 \%$ \\
\hline 5 (fatal or liver transplant) & 0 & $0 \%$ \\
\hline \multicolumn{3}{|l|}{ Causality scores } \\
\hline Definite & & 25 \\
\hline Highly likely & & 19 \\
\hline Probable & & 0 \\
\hline
\end{tabular}

Data not available on all 44 patients; number given in brackets.

${ }^{a}$ Chronicity defined as any abnormal of serum ALT, total bilirubin or alkaline phosphatase 6 mo after presentation.

${ }^{b}$ Only 36 subjects whose duration can be calculated with observed bilirubin level $<2.5$.

'Only 16 of the patients came for their 6-mo follow-up.

dPercentage of chronic patients at 6 mo followed up at 12 and 24 mo. clinical or laboratory differences in those that were heavy drinkers $(n=5)$ compared to the nonheavy alcohol consumers $(n=30)$. No one reported any pre-existing diabetes or chronic medical condition and only three had hypertension. One patient was HIV positive and three had established chronic HCV infection. The median time from the start of the bodybuilding supplement to onset of liver injury was 76 days, $9 \%$ occurring within the first $2-4$ weeks and $14 \%$ occurring after 24 weeks, although precise dates of starting and stopping the supplements were often lacking.

All patients presented with jaundice and most (84\%) with pruritus, strongly suggesting cholestatic liver injury. Ninety-eight per cent took an oral agent and one used self-administered intramuscular injections of reported testosterone-3-propionate, which had been purchased in Mexico without a prescription. None of the bodybuilding supplements taken by the patients were prescribed or supervised by a physician. All of them consumed the supplements to enhance physique or augment muscle mass, often in combination with an exercise and weight loss programme and with various nutritional supplements such as whey protein and creatine.

Table 2 includes results of initial laboratory tests obtained an average of 96 days after initiating bodybuilding supplements. The median initial serum ALT value was modestly elevated (169 U/L) with a wide range (61-1254 U/L) and over half had ALT levels >3 ULN. All patients presented with significant jaundice with a median total serum bilirubin value of $9.8 \mathrm{mg} / \mathrm{dL}$ (range, 4.1-45.7 mg/dL). Despite the clinical symptoms suggestive of a cholestatic pattern of liver injury, the initial median Alk Phos value was only $111 \mathrm{U} / \mathrm{L}$ (range, 53-461 U/L), with $70 \%$ of subjects having values within the local normal range. The median calculated $R$ value on presentation was 3.9 , only $29 \%$ presenting with a cholestatic pattern of liver injury $(R<2)$ and $40 \%$ presenting with a hepatocellular pattern $(R>5)$, despite the prominence of jaundice and pruritus. The elevated $R$ value was largely due to the normal or minimally elevated levels of Alk Phos, rather than high ALT values. One or more gamma-glutamyl transpeptidase (GGT) levels were available in 39 patients, but only two at onset, and only two had raised values, with the peak median GGT of $44.5 \mathrm{U} / \mathrm{L}$, with a range of 13-118. Elevated serum cholesterol (median of $202 \mathrm{mg} / \mathrm{dL}: \mathrm{n}=34$, range 62-630) and triglyceride (median $233 \mathrm{mg} / \mathrm{dL}: \mathrm{n}=36$, range 6.3-614) levels were also observed at onset. Twenty per cent of subjects underwent an endoscopic retrograde cholangiopancreatography (ERCP) or magnetic resonance cholangiopancreatography, and $59 \%$ had a liver biopsy as part of their initial evaluation.

\section{2 | Duration of injury and pattern of hepatic injury}

Jaundice was typically prolonged, the median time from onset to serum bilirubin falling below $2.5 \mathrm{mg} / \mathrm{dL}$ being slightly more than 3 months (94 days). Typically, serum aminotransferase levels fell rapidly during the course of the disease, the peak median ALT value (189 U/L) being minimally higher than the median value on 
presentation (169 U/L). In contrast, serum Alk Phos levels typically rose, the peak median value being twice that of the initial value (257 vs $111 \mathrm{U} / \mathrm{L}$ ). For these reasons, the $R$ value fell rapidly during the course of the injury to a median of less than 2 (cholestatic) within 2 weeks. Figure 1 illustrates the pattern of serum ALT, Alk Phos and bilirubin over time after enrollment based on available data. Despite the marked hyperbilirubinemia, signs of hepatic failure were uncommon with the median peak INR being 1.1 (range from 0.9 to 2.1) with only 6 greater than 1.5 . No patient developed ascites or hepatic encephalopathy. Several, however, developed renal insufficiency with serum creatinine levels rising above $1.5 \mathrm{mg} / \mathrm{dL}$ in six patients and above $2.5 \mathrm{mg} / \mathrm{dL}$ in 3 (peak values $2.7,3.2$ and $3.3 \mathrm{mg} / \mathrm{dL}$ ). Peak serum bilirubin levels in these three patients with renal insufficiency were all very high (46.7, 46.8 and $50.9 \mathrm{mg} / \mathrm{dL}$ ), and all patients had resolution of the renal insufficiency with recovery from the liver injury.

\section{3 | Liver histology}

Twenty-six patients underwent liver biopsies, of which 22 were available for independent review by the DILIN hepatic pathologist (DEK), who was masked to all clinical information. The median time from DILI onset to the liver biopsy was 15 days (range 1-68). The most common histologic pattern was a mixed hepatocellular and cholestatic injury (77\%), and only 4 (18\%) had acute cholestasis. Hepatocellular injury was typically spotty and mild, and no patient had bridging or confluent necrosis. Some degree of cholestasis was found in all biopsies but bile duct injury was found in only $14 \%$ without any bile duct loss. Steatosis was uncommon (10\%), and no patient had more than mild portal fibrosis. One case showed microscopic evidence suggestive of peliosis hepatis and nodular regeneration. Figure 2 illustrates some common histological features identified.

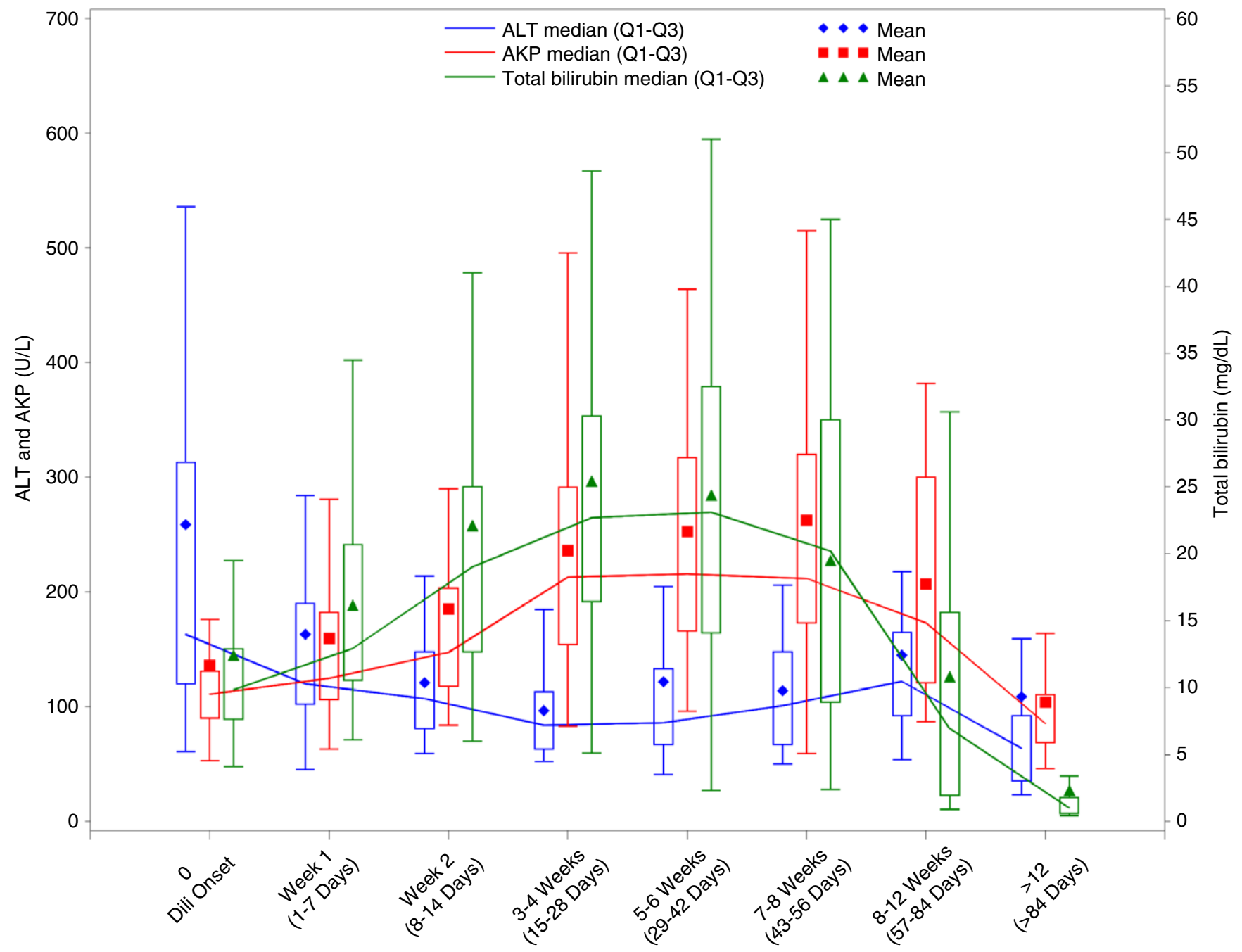

Weeks (Days) since Dili Onset

FIGURE 1 Median total bilirubin (mg/dL) ( $\mu \mathrm{mol}$ ) or Alk Phos (AKP) and ALT activity in U/L plotted over time in weeks after presentation. The lines connect the median values over time for each serum test. The numbers of subjects at different time period for ALT are as follows (with about \pm 1 for others): 44 at onset (day 0); 36 at week 1; 31 at week 2; 35 for week 3-4; 29 for week 5-6; 22 for week 7-8; 23 for week 8-12; 36 for $>12$ weeks. Mean values are depicted as symbols inside the box with the top and bottom of the box representing the 25 and 75 percentiles, respectively 

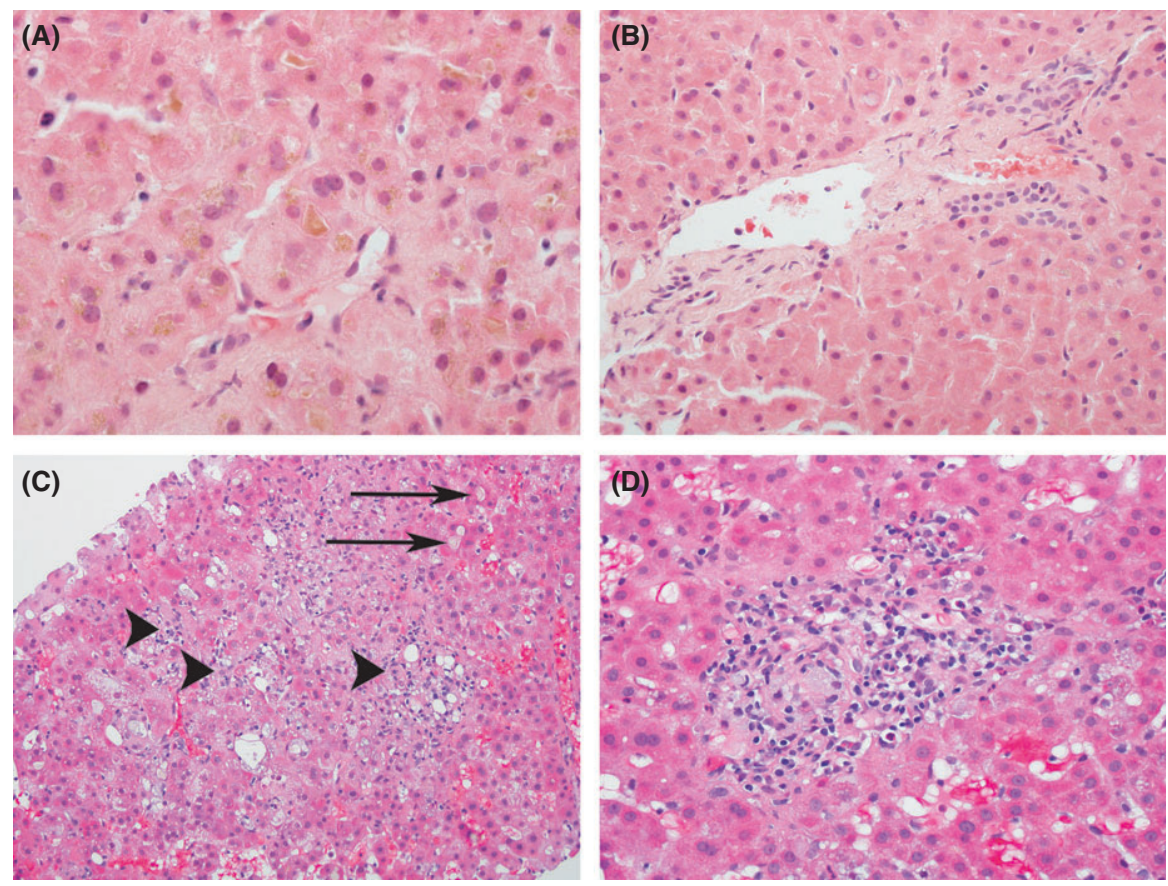

FIGURE 2 Histological findings in anabolic steroid DILI. Anabolic androgenic steroid liver injury is classically associated with bland cholestasis. There is canalicular and hepatocellular cholestasis with little or no lobular inflammation (A), and portal areas have a normal appearance without inflammation (B). (H\&E, 600x and 400x, respectively). Most of the cases in this report had mild degree of inflammation. Multiple small foci of lobular inflammation (arrowheads) are seen along with canalicular cholestasis (arrows) (C). The portal areas of this case also show inflammation (D)

\subsection{Causality assessment, severity of liver injury and chronicity}

Of the 44 cases, 25 were scored as definite and 19 as highly likely, while none were considered probable, reflecting the consistent phenotype and demographic characteristics of those with bodybuilding supplement-induced liver injury. The mean severity of illness was 3 with a range of 2-4 where 2 represented the presence of jaundice, 3 as jaundice with hospitalisation and 4 as severe injury with evidence of hepatic or another organ failure. ${ }^{13}$ Thirty-one subjects (71\%) were hospitalised for the acute liver injury (severity 3), but none died or underwent liver transplantation (severity 5) as was recently reported in another cohort. ${ }^{20}$

Of the 44 subjects with bodybuilding supplement-induced liver injury, only 16 (36\%) returned for their scheduled 6-month follow-up visit, of whom half had ALT greater than ULN. Twelve subjects returned at 12 months with 2 having ALT greater than ULN. In all cases, the serum enzyme elevations were mild and no patient was persistently jaundiced or symptomatic.

\subsection{Chemical analysis of bodybuilding supplement components}

Starting in 2009, the available incriminated herbal and dietary supplement products were submitted to a centralised repository, where they were catalogued and stored for future analysis. Thirty-three bodybuilding products were collected from 14 patients (subjects commonly consumed more than one bodybuilding supplement at a time) and were screened for anabolic steroids and related compounds. At least, one anabolic steroid was identified in 14 bodybuilding supplements provided by nine of the 14 patients but not in the 19 supplements from the remaining five. The labels often did not accurately reflect the chemical structure of the components as 17 of the 21 bodybuilding supplements contained anabolic steroids that were different from those listed on the label, and 12 of the steroids listed on the labels were not identified by chemical analysis. Only six anabolic steroids listed on the label were confirmed by chemical analysis. The anabolic steroids that were most frequently encountered were methyldrostanolone (55\%) followed by tetrahydrocorticosterone (44\%), 4-androstenedione (33\%) and DHEA (33\%). Listed in Table S1 are the case numbers, names of the primary bodybuilding supplements suspected to be the cause of the liver injury, whether the product label listed an anabolic steroid as an ingredient and, in 14 samples (nine subjects), whether an anabolic steroid was identified analytically. In some cases, none of the products contained detectable anabolic steroids or the product believed to contain one was not available for testing. Thirteen of the steroids identified were DEA controlled substances.

\subsection{Genetic association studies}

Twenty-five patients underwent WGS. Among the 22 patients of European-American ethnicity who underwent WGS, four were heterozygous (carriers) for rare missense variants in candidate genes previously reported to be associated with progressive familial intrahepatic cholestasis (PFIC) disorders: ATP8B1 (PFIC1), ABCB11 (BSEP) (PFIC2) or ABCB4 (MDR3) (PFIC3) (Table 3). Three additional patients, identified by exomic chip analysis, had variants in ATP8B1: two carried the D70N missense variant associated with PFIC/BRIC1 cases, which also showed some evidence of enrichment in our cases (case MAF (Minor allele frequency) $2.9 \%$ vs $0.4 \%$ in gnomAD controls, $P=0.04$ ) and one had the N45T missense variant, which has a frequency of $0.75 \%$ in European-American ancestry controls. Finally, a single subject had a rare variant in ABCB4, namely R590Q, which 
TAB LE 3 Rare functional variants identified in selected patients

\begin{tabular}{llllllll} 
Subject & $\begin{array}{l}\text { Genetic } \\
\text { ancestry }\end{array}$ & $\begin{array}{l}\text { Genetic } \\
\text { dataset }\end{array}$ & Gene & $\begin{array}{l}\text { Variant (amino } \\
\text { acid position) }\end{array}$ & Variant (chromosomal position) & $\begin{array}{l}\text { gnomad } \\
\text { MAF }^{21, a} \text { (\%) }\end{array}$ & $\begin{array}{l}\text { Polyphen Humdiv } \\
\text { Prediction }^{22}\end{array}$ \\
\hline 5 & Eur & Chip & ABCB11 & E297G & $2-169847329-T-C$ & 0.04 & Probably damaging \\
\hline 7 & Eur & Chip & ABCB11 & I206V & $2-169850388-T-C$ & 0.00 & Benign \\
\hline 11 & Eur & WGS & ATP8B1 & D70N & $18-55373793-C-T$ & 0.44 & Probably damaging \\
\hline 13 & Eur & WGS & ATP8B1 & N45T & $18-55398906-T-G$ & 0.75 & Possibly damaging \\
\hline 18 & Eur & WGS & ABCB4 & R590Q & 7-87060844-C-T & 0.68 & Probably damaging \\
\hline 30 & Eur & WGS & ATP8B1 & D70N & $18-55373793-C-T$ & 0.44 & Probably damaging \\
\hline 39 & Eur & Chip & ABCB11 & E297G & $2-169847329-T-C$ & 0.04 & Probably damaging \\
\hline 41 & AA & WGS & ABCB11 & V1112I & $2-169787252-C-T$ & 0.02 & Probably damaging \\
\hline
\end{tabular}

Eur indicates European, AA indicates African American; Chip indicates Illumina HumanExome chip, WGS indicates whole genome sequencing; ${ }^{\mathrm{M} M A F}$ is reported for non-Finnish Europeans for all cases except for the variant found in an African American patient, where the MAF reported is for Africans.

has a frequency of $0.68 \%$ in controls. When including the 12 patients of European-American ethnicity who had exome chip data available, three additional carriers of rare missense variants were identified, all in the ABCB11 gene. Two carried the E297G variant previously associated with PFIC2, which has a frequency of $0.04 \%$ in European ancestry controls, and one carried the 1206V variant, which has a frequency of $0.002 \%$ in controls. E297G variant in $A B C B 11$ is extremely rare in population controls of European ancestry (MAF 0.04\%) but was found in two of the 34 European ancestry cases (MAF 2.9\%; Fisher's exact test $P=0.0004$ ) and demonstrated a significant difference between our patients and the European ancestry controls in gnomAD. ${ }^{21}$ In addition, one of the five AfricanAmerican patients carried the variant $\mathrm{V} 1112 \mathrm{I}$ in $\mathrm{ABCB} 11$, which has a frequency of $0.02 \%$ in African ancestry controls. The non-European cases did not have any rare functional variants in any of these three genes identified by exome chip analysis.

To asses for overall enrichment of rare functional variants in these three genes in cases as compared to population controls, data from the gnomAD database were used to conservatively estimate that $12.3 \%$ of European ancestry population controls have a coding variant with a MAF below $1 \%$ in at least one of these genes, as compared to $20.6 \%$ in our cases. As 12 of our European ancestry cases were genotyped only for low-frequency coding variants by chip analysis (for included variants, see Table S2), some very rare variants may have been missed, but the frequency overall of cases with rare missense mutations in at least one of these genes was similar between our WGS cases (18\%) and our exome chip cases (25\%). When variants were removed as predicted by PolyPhen- 2 HumDiv ${ }^{22}$ to be benign, only $8.1 \%$ of the population controls were predicted to be carriers, whereas $17.6 \%$ of cases remained carriers. Almost $9 \%$ of our European ancestry cases had rare missense variants predicted to be probably damaging in ATP8B1 as compared to $4.3 \%$ of controls (8.9\% vs $5.6 \%$ when including benign variants), $5.8 \%$ in $A B C B 11$ compared to $1.0 \%$ in controls (8.9\% vs $3.3 \%$ with benign) and $2.9 \%$ in ABCB4 compared to $2.7 \%$ in controls (2.9\% vs $3.4 \%$ with benign). In the bodybuilding supplement cases, there was some clustering of variants in specific regions of $A B C B 11$ gene as compared to controls. The two $A B C B 11$ variants found in European ancestry cases, one of which was seen in two cases, were both in the $A B C$ transmembrane domain. In contrast, no more than $0.4 \%$ of European ancestry gno$\mathrm{mAD}$ controls have rare missense variants in this region. Similarly, the two ATP8B1 variants found in our cases, one of which was seen in two cases, were within 25 amino acids of each other; in contrast, no more than $2.5 \%$ of controls have rare missense variants within 25 amino acids of either of these variants (Figure S1). These findings suggest that genetic variants, particularly in $A B C B 11$, might predispose to bodybuilding supplement-associated cholestatic injury in some individuals, as has been postulated for cholestasis due to pharmaceutical agents. ${ }^{23}$

\section{4 | DISCUSSION}

\subsection{Clinical features of cholestatic liver injury due to anabolic steroids}

The clinical features of cholestatic liver injury in these 44 patients are consistent with prior observations of those exposed to anabolic steroids that include C-17 alpha alkylation. ${ }^{3,24}$ The next largest case series describes 25 cases of anabolic steroid-associated liver injury, predominately attributed to stanozolol. ${ }^{25}$ Other clinical descriptions consist of small case series with predominately oral agents. However, our study includes prospective data providing more detailed clinical course description and extensive genetic analysis in a majority of cases along with chemical analysis of bodybuilding supplements ingested, the latter carrying important regulatory implications.

All of our cases arose in men, typically young with few other medical problems. The vast majority admitted to drinking some alcohol in the past year, and we previously reported that heavy alcohol consumption was associated with exposure to bodybuilding supplements. ${ }^{19}$ As compared to the subjects with bodybuilding supplement-associated DILI, the entire cohort of subjects enrolled into the DILIN with causality score of probably or greater, reported that only $50 \%$ had some alcohol consumption in the past year and of those that completed the alcohol survey, less than $23 \%$ were heavy drinkers as previously defined. ${ }^{19}$ Subjects with bodybuilding 
supplement-associated DILI commonly presented with pruritus and reported weight loss during their cholestatic period. The rapid reduction of ALT followed by increasing values of the total serum bilirubin and Alk Phos as the injury progressed suggests that hepatocellular injury is an early event in bodybuilding supplement-induced liver injury despite the cholestatic clinical presentation. Transient elevations in aminotransferase levels have been reported during acute biliary obstruction due to stones or as the initial presentation of intrahepatic cholestasis of pregnancy. ${ }^{26,27}$ There are also other case reports of elevation of the ALT and increased $R$ value in anabolic steroid-associated liver injury. ${ }^{25,28}$ In addition, GGT levels, when obtained, were rarely elevated for unclear reason. Thus, the presenting clinical features of anabolic steroid-induced cholestasis were quite distinct leading to the high degree of certainty in their causality scores. In addition to the clinical presentation, exposure to anabolic steroids can also be confirmed by either the chemical detection of the anabolic steroid, typically in the urine or offending drug in the serum such as C-19 aromatase inhibitors as well as the physiological response to exogenously consumed anabolic steroid. These studies include the following: decrease in Follicle-stimulating hormone and Luteinizing hormone serum levels, changes in the epi-testosterone to testosterone ratio or in the $\mathrm{C}_{13} / \mathrm{C}_{12}$ ratio of androgenic steroids in the urine. ${ }^{29,30}$

With regard to renal insufficiency (as defined by serum creatinine $>1.5 \mathrm{mg} / \mathrm{dL}$ ), only six patients were affected and of these, only three had creatinine persistently elevated above $2.5 \mathrm{mg} / \mathrm{dL}$. The mechanism for renal injury during severe and prolonged cholestasis is unknown, but the anabolic steroid methasteron has been previously reported to be associated with renal injury, suggesting that renal injury may be associated with a specific chemical structure of the anabolic steroid. ${ }^{31}$ Alternatively, elevated serum bile salts have also been implicated in causing renal injury. ${ }^{32}$ Fortunately, none of the patients died or required liver transplantation. However, $16 \%$ of the patients were treated with a trial of corticosteroids, with little or no apparent clinical response, although this was not formally assessed. In addition, almost half were enrolled on Ursodiol therapy, presumably for cholestatic symptoms. Follow-up was difficult with these patients as only $36 \%$ returned for a 6-month follow-up, a rate far lower than the average patient enrolled in DILIN (80\%), probably reflecting the background good health of these patients and the illicit nature of the bodybuilding supplement use. None developed chronic cholestatic liver injury, vanishing bile duct syndrome ${ }^{33}$ or liver failure requiring liver transplantation. There was eventual resolution of all liver tests in those that were followed for greater than 6 months.

\section{2 | Liver biopsy findings}

All liver biopsies had histological features of profound intrahepatic cholestasis, without evidence of duct injury or loss. A mild hepatitis was noted without fibrosis, as has been previously reported. ${ }^{28}$ The liver histology features noted close to the time of elevated serum aminotransferases were not significantly different from those seen when the $R<5$, although there was a trend to an increased presence of hepatocyte apoptosis in the former. As none of the biopsies revealed alternative diagnoses, a liver biopsy may not be necessary when the distinct clinical presentation is evident in a young male and exposure history is consistent.

\section{3 | Genetic analysis}

The prolonged cholestasis with a normal GGT level, when tested, is clinically reminiscent of patients who present with BRIC due to genetic variants in ATP8B1, the $P$ type ATPase responsible for PFIC1 and BRIC-1, or ABCB11, the bile salt export protein responsible for PFIC2 or BRIC2. Prior genetic analysis of individuals with anabolic steroid-associated cholestatic liver injury with normal GGT identified heterozygous mutations in $\mathrm{ABCB} 11$ and 2 bp deletion in exon 1 of PXR. ${ }^{11,12}$ Thus, a concerted effort was made to identify genetic mutations using WGS and chip-based discovery. The majority of patients with cholestasis due to anabolic steroids do not have causal mutations in the genes ATP8B1, $A B C B 11$ (BSEP) or $A B C B 4$ (MDR3). Of note, more variants were found in the first $A B C$ membrane region of $A B C B 11$ and near the $N$ terminus of ATP8B1 as compared to controls (Figure S1). This trend will require confirmation. There were some limitations as 16 patients were genotyped with an exome chip, in which case rare variants may not be identified. For example, two of the variants found in genome sequencing were not evaluated on the exome chip. Because $12 \%$ of controls had similar variants in these genes, the proportion of cholestatic cases genetically explained is likely to be far lower than the maximum estimate. Therefore, mutations in these three candidate genes can only account for a maximum of $20 \%$ of our cases. As only $20 \%$ at most had genetic variants in genes responsible for rare genetic cholestatic syndrome liver injury may be due to unknown hepatic transporters either at the canalicular or sinusoidal domain of hepatocytes, or variants in other genes. ${ }^{34}$ Other possible mechanism may include secondary metabolic effects of anabolic steroids, such as physical chemical changes in membrane fluidity due to modification of the lipid composition at the canalicular domain or a direct physical chemical interaction of the anabolic steroids on membrane or biliary transporters' function.

\subsection{Chemical analysis of bodybuilding supplements}

As previously reported, bodybuilding supplement product labels correlated poorly with the chemical analysis, and the steroids species that were found were often not on the label. ${ }^{35}$ The presence of anabolic steroids in bodybuilding supplement samples, which are currently listed as controlled substances (https://www.deadiversion. usdoj.gov/21cfr/cfr/1300/1300_01.htm), illustrates that these DEA controlled steroids are easily available for purchase. Although anabolic steroids were not identified in samples from five patients, not all bodybuilding supplement samples were available for analysis. In addition, the common phenotypic presentation of these patients without anabolic steroids detected in these supplements strongly 
suggests exposure. Our data are particularly pertinent in the light of the increasing use of anabolic steroids in the adolescent population. A recent survey revealed that up to $1 \%$ of adolescent males and $0.1 \%$ of adolescent females have been exposed to anabolic steroids in high school with an estimated prevalence of use by 2.94 million Americans. ${ }^{9}$ Our chemical analysis confirmed identification of controlled steroids, and there was a poor correlation between label and actual content in bodybuilding supplements. These data along with others ${ }^{35}$ suggest that greater regulatory oversight is required to protect the general public.

Strengths of our study include the prospective enrollment of consecutive cases, high causality scores and extensive phenotyping including liver biopsies in over $50 \%$ of the cases. Available bodybuilding supplement samples were comprehensively analysed, and the chemical structure of the anabolic steroid identified in the vast majority. In this same cohort, genetic variants were sought using either WGS or chip-based discovery, which included known variants in PFIC genes. Weaknesses in our study were the retrieval of bodybuilding supplement agents in only a third of the subjects, inability to demonstrate a clear dose response to liver injury and inadequate follow-up due to poor subject compliance.

In conclusion, patients with liver injury due to bodybuilding supplements presented with a uniform and distinctive pattern of marked cholestatic liver injury with pruritus, that was often intractable, prolonged and accompanied by disability and weight loss. Liver biopsy failed to identify another cause suggesting that the clinical diagnosis of severe cholestasis with minimal elevation of liver tests may be sufficient to make the diagnosis. As our patient population was predominately young males, bodybuilding supplement use with likely anabolic steroid exposure should be strongly considered in the appropriate patient population who present with new onset jaundice together with mild elevations in the serum aminotransferase levels. This may be confirmed by direct testing for the steroid or the physiological consequences of exposure to anabolic androgens as described.

\section{ACKNOWLEDGEMENT}

Declaration of personal interests: All authors declared that they have no conflict of interest. Dr Chalasani has ongoing consulting activities (or had in preceding 12 months) with NuSirt, Abbvie, Eli Lilly, Afimmune (DS Biopharma), Tobira (Allergan), Madrigal, Shire, Cempra, Ardelyx, Gen Fit and Amarin. These consulting activities are generally in the areas of nonalcoholic fatty liver disease and drug hepatotoxicity. Dr Chalasani receives research grant support from Intercept, Lilly, Gilead, Galectin Therapeutics and Cumberland where his institution receives the funding. Over the last decade, $\mathrm{Dr}$ Chalasani has served as a paid consultant to more than 30 pharmaceutical companies and these outside activities have regularly been disclosed to his institutional authorities. Dr Fontana has received research support from BMS, Janssen, and Gilead and served as a consultant to Alynam. Dr Bonkovsky serves as consultant to Alnylam Pharma, Blue, Mitsubishi-Tanabe, Moderna, Recordati rare
Chemicals, and Stoke. He receives support for clinical studies from Alnylam and Gilead Sciences in the area of porphyrias. Dr Bonkovsky serves as a member of the Medical and Scientific Advisory Boards of the American Porphyria Foundation and the Iron Disorders Institute. Dr Seeff is presently a consultant to Abbvie and Intercept and was a consultant to Cempra Drs Barnhart, Gu, Stolz, Hayashi, Navarro, Serrano, Kleiner, Cirruli, Avula, and Hoofnagle have no conflicts of interest to disclose.

\section{AUTHORSHIP}

Guarantor of the article: A Stolz.

Author contributions: All of the authors contributed to study concept and design, acquisition of data, analysis of data, and critical review of the early and final manuscript drafts. Statistical analysis was performed by (Barnhart, Gu), liver histology by Kleiner, acquisition and chemical analysis of bodybuilding supplements by (Bonkovsky, Vega, Avula and Khan) and genetic analysis by Cirulli. All authors approved the final version of the manuscript.

\section{ORCID}

Andrew Stolz (D) https://orcid.org/0000-0002-1443-9351

Naga P. Chalasani iD https://orcid.org/0000-0003-4082-3178

\section{REFERENCES}

1. Shahidi NT. A review of the chemistry, biological action, and clinical applications of anabolic-androgenic steroids. Clin Ther. 2001;23: 1355-1390.

2. Bagatell CJ, Bremner WJ. Androgens in men-uses and abuses. N Engl J Med. 1996;334:707-714.

3. Ishak KG, Zimmerman HJ. Hepatotoxic effects of the anabolic/androgenic steroids. Semin Liver Dis. 1987;7:230-236.

4. Bagheri SA, Boyer JL. Peliosis hepatis associated with androgenic-anabolic steroid therapy. A severe form of hepatic injury. Ann Intern Med. 1974;81:610-618.

5. Soe KL, Soe M, Gluud C. Liver pathology associated with the use of anabolic-androgenic steroids. Liver. 1992;12:73-79.

6. Barceloux DG, Palmer RB. Anabolic-androgenic steroids. Dis Mon. 2013;59:226-248.

7. National Library of Medicine. Anabolic Steroids. https://livertox.nih. gov/AndrogenicSteroids.html Accessed October 1, 2018.

8. Velazquez I, Alter BP. Androgens and liver tumors: Fanconi's anemia and non-Fanconi's conditions. Am J Hematol. 2004;77:257-267.

9. Pope HG Jr, Kanayama G, Athey A, Ryan E, Hudson Jl, Baggish A. The lifetime prevalence of anabolic-androgenic steroid use and dependence in Americans: current best estimates. Am J Addict. 2014;23:371-377.

10. Kanayama G, Pope HG Jr. History and epidemiology of anabolic androgens in athletes and non-athletes. Mol Cell Endocrinol. 2018;464:4-13.

11. El Sherrif Y, Potts JR, Howard MR, et al. Hepatotoxicity from anabolic androgenic steroids marketed as dietary supplements: contribution from ATP8B1/ABCB11 mutations? Liver Int. 2013;33:1266-1270.

12. Liebe R, Krawczyk M, Raszeja-Wyszomirska J, et al. Heterozygous inactivation of the nuclear receptor PXR/NR1/2 in a patient with anabolic steroid-induced intrahepatic cholestasis. Hepat Mon. 2016; 16:e35953. 
13. Chalasani N, Bonkovsky HL, Fontana R, et al. Features and outcomes of 899 Patients with drug-induced liver injury: the DILIN prospective study. Gastroenterology. 2015;148:1340-1352 e1347.

14. Fontana RJ, Watkins PB, Bonkovsky HL, et al. Drug-induced liver injury network (DILIN) prospective study. Drug Saf. 2009;32:55-68.

15. Navarro VJ, Barnhart H, Bonkovsky HL, et al. Liver injury from herbals and dietary supplements in the U.S. Drug-Induced Liver Injury Network. Hepatology. 2014;60:1399-1408.

16. Hayashi PH, Barnhart HX, Fontana RJ, et al. Reliability of causality assessment for drug, herbal and dietary supplement hepatotoxicity in the Drug-Induced Liver Injury Network (DILIN). Liver Int. 2014;35:1623-1632.

17. Kleiner DE, Chalasani NP, Lee WM, et al. Hepatic histological findings in suspected drug-induced liver injury: systematic evaluation and clinical associations. Hepatology. 2013;59:661-670.

18. Jacquemin E. Progressive familial intrahepatic cholestasis. Clin Res Hepatol Gastroenterol. 2012;36(Suppl. 1):S26-S35.

19. Dakhoul L, Ghabril M, Gu J, et al. Heavy consumption of alcohol is not associated with worse outcomes in patients with idiosyncratic drug-induced liver injury compared to non-drinkers. Clin Gastroenterol Hepatol. 2018;16:722-729 e722.

20. Medina-Caliz I, Garcia-Cortes M, Gonzalez-Jimenez A, et al. Herbal and dietary supplement-induced liver injuries in the Spanish DILI registry. Clin Gastroenterol Hepatol. 2018;16:1495-1502.

21. Lek M, Karczewski KJ, Minikel EV, et al. Analysis of protein-coding genetic variation in 60,706 humans. Nature. 2016;536:285-291.

22. Adzhubei I, Jordan DM, Sunyaev SR. Predicting functional effect of human missense mutations using PolyPhen-2. Current protocols in human genetics/editorial board, Jonathan L Haines [et al.]. 2013; Chapter 7: Unit7 20.

23. Lang C, Meier Y, Stieger B, et al. Mutations and polymorphisms in the bile salt export pump and the multidrug resistance protein 3 associated with drug-induced liver injury. Pharmacogenet Genomics. 2007; 17:47-60.

24. Schaffner F, Popper $\mathrm{H}$, Chesrow E. Cholestasis produced by the administration of norethandrolone. Am J Med. 1959;26:249-254.

25. Robles-Diaz M, Gonzalez-Jimenez A, Medina-Caliz I, et al. Distinct phenotype of hepatotoxicity associated with illicit use of anabolic androgenic steroids. Aliment Pharmacol Ther. 2015;41:116-125.

26. Nathwani RA, Kumar SR, Reynolds TB, Kaplowitz N. Marked elevation in serum transaminases: an atypical presentation of choledocholithiasis. Am J Gastroenterol. 2005;100:295-298.
27. Jurate K, Rimantas Z, Jolanta S, Vladas G, Limas K. Sensitivity and specificity of biochemical tests for diagnosis of intrahepatic cholestasis of pregnancy. Ann Hepatol. 2017;16:569-573.

28. Stimac D, Milic S, Dintinjana RD, Kovac D, Ristic S. Androgenic/Anabolic steroid-induced toxic hepatitis. J Clin Gastroenterol. 2002;35: 350-352.

29. Anawalt BD. Detection of anabolic androgenic steroid use by elite athletes and by members of the general public. Mol Cell Endocrinol. 2018;464:21-27.

30. Nieschlag E, Vorona E. Mechanisms in endocrinology: medical consequences of doping with anabolic androgenic steroids: effects on reproductive functions. Eur J Endocrinol. 2015;173:R47-R58.

31. Shah NL, Zacharias I, Khettry U, Afdhal N, Gordon FD. Methasteronassociated cholestatic liver injury: clinicopathologic findings in 5 cases. Clin Gastroenterol Hepatol. 2008;6:255-258.

32. Alkhunaizi AM, EITigani MA, Rabah RS, Nasr SH. Acute bile nephropathy secondary to anabolic steroids. Clin Nephrol. 2016;85: 121-126.

33. Bonkovsky HL, Kleiner DE, Gu J, et al. Clinical presentations and outcomes of bile duct loss caused by drugs and herbal and dietary supplements. Hepatology. 2017;65:1267-1277.

34. Boyer JL. Bile formation and secretion. Compr Physiol. 2013;3:1035-1078.

35. Abbate V, Kicman AT, Evans-Brown M, et al. Anabolic steroids detected in bodybuilding dietary supplements - a significant risk to public health. Drug Test Anal. 2015;7:609-618.

\section{SUPPORTING INFORMATION}

Additional supporting information will be found online in the Supporting Information section at the end of the article.

How to cite this article: Stolz A, Navarro V, Hayashi PH, et al. Severe and protracted cholestasis in 44 young men taking bodybuilding supplements: assessment of genetic, clinical and chemical risk factors. Aliment Pharmacol Ther. 2019;49:1195-1204. https://doi.org/10.1111/apt.15211 